Working Paper

Business Economic Series

WP. 14-02

ISSN 1989-8843
Instituto para el Desarrollo Empresarial. Universidad Carlos III de Madrid

C/ Madrid, 126

28903 Getafe Madrid (Spain)

FAX (34-91)6249607

\title{
Information Disclosure in Optimal Auctions
}

\author{
Juan-José Ganuza † \\ Universitat Pompeu Fabra, Barcelona \\ and \\ Jose Penalva $\ddagger$ \\ Universidad Carlos III, Madrid
}

March, 2014

† Department of Economics, Universitat Pompeu Fabra. E-mail: juanjo.ganuza@upf.edu.

‡ Department of Business, Universidad Carlos III. E-mail: jpenalva@econ.uc3m.es. 


\title{
Information Disclosure in Optimal Auctions.*
}

\author{
Juan-José Ganuza ${ }^{\dagger}$ and Jose Penalva ${ }^{\ddagger}$
}

MARCH, 2014.

\begin{abstract}
A celebrated result in auction theory is that the optimal reserve price in the standard private value setting does not depend on the number of bidders. We modify the framework by considering that the seller controls the accuracy with which bidders learn their valuations, and show that in such a case, the greater the number of bidders the more restrictive the reserve price. We also show that the auctioneer provides more information when using an optimal auction mechanism than when the object is always sold.
\end{abstract}

KeYwords: Auctions, Private Values, Information Disclosure.

JEL classification numbers: C72, D44, D82, D83.

\footnotetext{
${ }^{*}$ We are grateful for comments by Preston McAfee the seminar participants at UPF (Barcelona). The usual disclaimers apply.

${ }^{\dagger}$ Department of Economics, Universitat Pompeu Fabra. E-mail: juanjo.ganuza@upf.edu. Juan-José Ganuza gratefully acknowledges the support of the Barcelona GSE Research, the government of Catalonia, and the Spanish Ministry of Education and Science Through Project ECO 2011-28965.

${ }^{\ddagger}$ Department of Business, Universidad Carlos III. E-mail: jpenalva@econ.uc3m.es. Jose Penalva acknowledges the support of the Spanish Ministry of Education and Science Through Project ECO 2012-36559.
} 


\section{INTRODUCTION}

We analyze a situation in which the seller of an object controls the accuracy with which $N$ risk neutral bidders learn their private valuations. The object is sold using an optimal auction with reserve price. A celebrated result in auction theory is that the optimal reserve price depends on the distribution of bidder valuations but not on the number of bidders. In our framework, where the auctioneer chooses how much information to disclose, competition between bidders (captured by the number of bidders, $N$ ) affects the incentives of the auctioneer to disclose information, which in turn affects the distribution of bidder valuations, and hence, the reserve price. What we find is that a greater number of bidders, increases the optimal amount of information disclosed, which leads to a more restrictive reserve price.

This paper contributes to two different branches of the literature. Jullien and Mariotti (2006) and Cai, Riley, and Yi (2007) show that in an affiliated value setting the auctioneer uses the reserve price to signal his valuation. In that setting, the number of bidders affects the incentives of the seller to signal through the reserve price. In our private value setting, the seller's valuation is not relevant for bidders' decision problems and the reserve price has no informational content. We also contribute to the literature that analyzes the auctioneer's incentives to disclose information in private value settings that usually assumes that the object is always sold (see for example, Ganuza (2004), Board (2009), Ganuza and Penalva (2010) and Hummel and McAfee (2012)). ${ }^{1}$ We show that the auctioneer provides more information when using an optimal mechanism, an auction with reserve price. Providing information to bidders has a positive effect, it increases the efficiency of the allocation (and bidders' willingness to pay), but it also has a negative effect, it increases bidders' informational rents. ${ }^{2}$ As the reserve price reduces bidder informational rents, it increases

\footnotetext{
${ }^{1}$ All these papers, as well as the current one, assume that the auctioneer chooses from a given family of indexed information structures. Bergeman and Pesendorfer (2007) studied the joint problem of costlessly designing bidders' private information and the corresponding optimal mechanism. While they consider optimal mechanisms, in their setting it is very difficult to study the effects of competition on information provision as the resulting (optimal) information structures are not ordered in terms of informativeness.

${ }^{2}$ In an alternative setting, Eső and Szentes (2007) shows that if the auctioneer can commit to the informativeness of the signals provided and charge bidders for it, then the auctioneer can extract all the informational rents ex-ante, and the tradeoff between efficiency and bidders' rents vanishes.
} 
the incentives of the auctioneer to disclose more information. This result is in line with the one in Hummel and McAfee (2012), where in a setting of all-or-nothing costless information disclosure, the minimum number of bidders required for the optimality of full information provision is lower with a reserve price than without one.

\section{The Model}

An auctioneer wishes to sell a single object to one of $N$ risk-neutral bidders using a standard auction, such as a second price sealed auction. ${ }^{3}$ The auctioneer's valuation of the object is 0. Bidder valuations, $v_{i}, i \in\{1,2, \ldots, N\}$, are identically and independently distributed with cumulative distribution $F(v)$, support on $[0,1]$, a strict positive and differentiable density, $f(v)$, on $[0,1]$ and mean $v_{m} . F(v)$ satisfies the monotone hazard rate assumption, which implies that the virtual valuation function, $J(v)=v-\frac{1-F(v)}{f(v)}$, is increasing in $v$.

Bidders do not know their valuations. Before the auction, the auctioneer discloses information which generates a private signal for each bidder, $x_{i}$. The auctioneer chooses how much information to make available by determining the precision of bidders' signals, denoted by $\delta$, which is publicly observed. For the auctioneer obtaining and transmitting information is costly. In particular, creating $N$ signals with precision $\delta$ has a cost $C(\delta)$, where $C^{\prime}(\delta)>0, C^{\prime \prime}(\delta)>0$,and $C^{\prime}(0)=0$.

Finally, bidders, after updating their expected valuations using their private signals, submit offers to the auctioneer, and the auction takes place.

Summarizing, the time sequence is as follows:

1. The auctioneer, knowing the number of bidders, $N$, decides how much information to disclose to the market by choosing $\delta$ at a cost $C(\delta)$.

2. Given $\delta$, each bidder receives a private signal $x_{i}$. Bidders update their valuations of the object using $\delta$ and $x_{i}$.

\footnotetext{
${ }^{3}$ The Revenue Equivalence Theorem applies in our setting. For concreteness, it may be useful to think of the auctioneer as using a second price sealed auction although all our results hold for any standard auction.
} 
3. The auction takes place and the object is awarded.

We solve the model by backward induction.

\subsection{Updating bidders' valuations.}

We focus on a particular family of information structures ordered in terms of informativeness by $\delta$. For given $\delta$, agent $i$ receives a signal, $x_{i}$, where this signal is known to be equal to the bidder's true private valuation, $x_{i}=v_{i}$, with probability $\delta$, while with the complementary probability, $x_{i}$ is pure noise with distribution $F(v)$. Thus, independently of the accuracy of the signal, $x_{i}$ is distributed according to $F(v)$. This implies that the expected valuation of the object for a bidder $i$ who receives a signal $x_{i}$ when precision is $\delta, v_{i}^{E}\left(x_{i}, \delta\right)$ is equal to:

$$
\begin{aligned}
v_{i}^{E}\left(x_{i}, \delta\right) & =E\left[v_{i} \mid x_{i}, \delta\right] \\
& =x_{i} \delta+(1-\delta) v_{m}
\end{aligned}
$$

From the point of view of the auctioneer, who does not observe signal realizations, the setup is still one of standard private values, where each bidder's private valuation, $v_{i}^{E}$, is an independent draw from a new distribution, $G\left(v^{E}, \delta\right)$. The distribution of updated expected valuations is obtained from $F(v)$ using a simple change of variable.

$$
G\left(v^{E}, \delta\right)=F\left(\frac{v^{E}-(1-\delta) v_{m}}{\delta}\right) .
$$

The main properties of $G(\cdot, \delta)$ are:

Lemma 1 (a) For all $\delta \in(0,1)$, the distribution function $G(v, \delta)$ induces a virtual valuation function, $v-\frac{1-G(v, \delta)}{g(v, \delta)}$, that is increasing in $v$. (b) For all $\delta \in(0,1)$, and $\delta>\delta^{\prime}, G\left(v, \delta^{\prime}\right)$ dominates $G(v, \delta)$, in the sense of second degree stochastic dominance (and in terms of the dispersive order).

The fact that $G$ preserves the monotonicity of the virtual valuation function allows us to focus our analysis wlog on standard auction mechanisms such as the second price auction. 
Let the expected revenue of the auctioneer when the object is always sold be denoted by $\pi(\delta, N)$ where $\pi$ is defined by the following equation:

$$
\pi(\delta, N)=\int_{(1-\delta) v_{m}}^{\delta+(1-\delta) v_{m}}\left[v_{1}^{E}-\frac{1-G\left(v_{1}^{E}, \delta\right)}{g\left(v_{1}^{E}, \delta\right)}\right] m_{g}\left(v_{1}^{E}, \delta\right) d v_{1}^{E}-C(\delta),
$$

where $m_{g}\left(v_{1}^{E}, \delta\right)$, is the density function of $v_{1}^{E}$ which is the maximum of $N$ independent draws from $G\left(v^{E}, \delta\right)$. Then, the auctioneer's optimal information disclosure decision is obtained as the solution to the following problem:

$$
\delta^{*} \in \underset{\delta}{\operatorname{argmax}} \pi(\delta, N)
$$

From existing results (see Ganuza and Penalva (2010) and Simon (2009)) we obtain the following proposition.

Proposition 1 There exist $N_{0}$ such that for $N \leq N_{0}$ it is not optimal to reveal any information, while for $N>N_{0}$ it is optimal to reveal some amount of information, $\delta^{*}(N)>0 .{ }^{4}$ The optimal amount of information (when the object is always sold) $\delta^{*}$ is increasing in the number of bidders, $N{ }^{5}$

To illustrate this result consider a special case where the distribution of bidder valuations is uniform on $[0,1]$, and the cost of obtaining and transmitting information is quadratic, i.e $C(\delta)=\theta \delta^{2}$. For given $\delta$, the distribution of expected valuations, $v^{E}$ is uniformly distributed over the interval, $\left[\frac{(1-\delta)}{2}, \frac{(1+\delta)}{2}\right]$. The optimal amount of information is $\delta^{*}=\frac{1}{2 \theta}\left(\frac{N-1}{N+1}-\frac{1}{2}\right)$ if $N$ is larger than 3 , otherwise it is 0 . Notice, the optimal amount of information, $\delta^{*}(N)$, is strictly increasing for $N>3$.

\footnotetext{
${ }^{4}$ In a costless information setting, Hummel and McAfee (2012) shows that $N_{0}$ is 3 or 4 . Simon (2009) do not impose the monotone hazard rate assumption, and using an example based on power distribution $F(x)=x^{\beta}$ proves that $N_{0}$ may be unbounded.

${ }^{5}$ As $\delta^{*}$ may not be a singleton, this statement as well as further comparative statics results below, should be interpreted in the sense of Veinott's strong set order: $\delta_{N+1}^{*} \geq \delta_{N}^{*}$ iff $\forall \delta \in \delta_{N+1}^{*}, \delta^{\prime} \in \delta_{N}^{*}, \max \left\{\delta, \delta^{\prime}\right\} \in \delta_{N+1}^{*}$ and $\min \left\{\delta, \delta^{\prime}\right\} \in \delta_{N}^{*}$
} 


\section{Optimal Mechanism: Reserve Price.}

Given the distribution of expected valuations $G(v, \delta)$, the optimal reserve price, denoted by $v^{R P}$, is given by the following expression:

$$
v^{R P}-\frac{1-G\left(v^{R P}, \delta\right)}{g\left(v^{R P}, \delta\right)}=0,
$$

where the left hand side is the virtual valuation of bidders and the right hand side, is the auctioneer's valuation of the object, which is 0 . Using the relationship between $G$ and $F$, the optimal reserve price can be characterized using the virtual valuation function of the prior distribution, $J(v)=v-\frac{1-F(v)}{f(v)}$, and a cutoff value of the signal, $x^{R P}:$

Lemma 2 The optimal reserve price is $v^{R P}=x^{R P} \delta+(1-\delta) v_{m}$ where

$$
x^{R P}=\left\{\begin{array}{cc}
J^{-1}\left(-\frac{(1-\delta)}{\delta} v_{m}\right) & \text { if } J(0)<-\frac{(1-\delta)}{\delta} v_{m} \\
0 & \text { Otherwise }
\end{array}\right.
$$

This result determines the relationship between a given level of precision, $\delta$, and the optimal reserve price.

Proposition 2 There exists a level of information $\bar{\delta}=\frac{v_{m}}{v_{m}-J(0)} \in[0,1]$ such that: (i) if the auctioneer sets $\delta \leq \bar{\delta}$, the optimal selling strategy is not to use a reserve price, and (ii) if the auctioneer sets $\delta>\bar{\delta}, x^{R P}=J^{-1}\left(-\frac{(1-\delta)}{\delta} v_{m}\right)$ and $x^{R P}$ is increasing in $\delta$. (iii) The probability that an individual bidder has an expected valuation lower than the optimal reserve price, $G\left(v^{R P}, \delta\right)=$ $F\left(x^{R P}\right)$ is increasing in $\delta$.

The intuition behind these results is based on the following two facts: (a) the informational rents are increasing in the amount of information provided; (b) the reserve price is a seller's tool to reduce informational rents. Putting these together, the greater the amount of information provided by the seller, the larger the informational rents, and the larger the seller's incentives to reduce such rents by increasing the reserve price relative to the distribution of expected valuations. 
Having determined the auctioneer's optimal selling strategy we now analyze the auctioneer's information disclosure problem. Let the auctioneer's expected revenue for disclosing information $\delta$ while using the optimal reserve price, $v^{R P}(\delta)$, is denoted by $\hat{\pi}(\delta, N)$, where

$$
\hat{\pi}(\delta, N)=\int_{v^{R P}(\delta)}^{\delta+(1-\delta) v_{m}}\left[v_{1}^{E}-\frac{1-G\left(v_{1}^{E}, \delta\right)}{g\left(v_{1}^{E}, \delta\right)}\right] m_{g}\left(v_{1}^{E}, \delta\right) d v_{1}^{E}-C(\delta) .
$$

Then, the optimal choice of precision when using an optimal mechanism, $\widehat{\delta}^{*}$, is

$$
\widehat{\delta}^{*} \in \underset{\delta}{\operatorname{argmax}} \hat{\pi}(\delta, N)
$$

The next proposition compares $\widehat{\delta}^{*}$ with the optimal one chosen when the object is always sold, $\delta^{*}$.

Proposition 3 When the auctioneer uses an optimal reserve price he provides more information than when the object is always sold, $\widehat{\delta}^{*} \geq \delta^{*}$.

As the reserve price reduces bidder rents, having a reserve price weakens the trade-off between efficiency and bidder rents, increasing the auctioneer's incentives to provide more information. We now look at the effect of greater competition $(N)$ on $\widehat{\delta}^{*}$.

Proposition 4 i) There exist $\widehat{N}_{0}$ such that for $N \leq \widehat{N}_{0}$ it is not optimal to reveal any information, while for $N>\widehat{N}_{0}$ it is optimal to reveal some amount of information, $\widehat{\delta}^{*}(N)>0$. The optimal amount of information (when the object is always sold) $\widehat{\delta}^{*}$ is increasing in the number of bidders, $N$.

ii) When the auctioneer uses an optimal reserve price he discloses some information with less competition than when the object is always sold, $\widehat{N}_{0} \leq N_{0}$.

As in the case without a reserve price (Proposition 4 ), greater competition between bidders $(N)$ reduces informational rents and increases the incentives of the auctioneer to provide information. Part ii) follows directly from the fact that for all $N$, it is more profitable to give information when using a reserve price than when not using one. Then, if the auctioneer wants to give some 
information when not using a reserve price, he will necessarily also want to give information when using one.

Finally, Proposition 2 and Proposition 4 jointly imply that a higher number of bidders leads the auctioneer to optimally set a more restrictive reserve price-in the sense that for any bidder, the probability of having a valuation higher than the reserve price is lower.

COROLlary 5 The probability that any individual bidder's valuation is below the optimal reserve price is weakly increasing in $N$.

Intuitively, more competition increases informational rents and hence the incentives of the auctioneer to use a reserve price to control those rents, which he does by making the standard for participating in the auction tougher.

\subsection{Example}

We continue with the previous example: uniformly distributed valuations and quadratic costs. The virtual valuation function of the prior distribution is, $J(x)=2 x-1$ and $\bar{\delta}=\frac{v_{m}}{v_{m}-J(0)}=\frac{1}{3}$. Therefore, (i) If $\delta<\frac{1}{3}$ the optimal selling strategy is not to use a reserve price. (ii) If $\delta>\frac{1}{3}$, $J\left(x^{R P}\right)=-\frac{(1-\delta)}{2 \delta} \Longrightarrow x^{R P}=\frac{3}{4}-\frac{1}{4 \delta}$, so that only bidders with signals greater than $\frac{3}{4}-\frac{1}{4 \delta}$ will participate in the auction. Hence, in this case, the optimal reserve price in terms of expected valuations is $v^{R P}=x^{R P} \delta+(1-\delta) \frac{1}{2}=\frac{1+\delta}{4}$, which is increasing in the amount of information. The optimal amount of information is implicitly defined by the following expression

$$
\int_{\frac{3}{4}-\frac{1}{4 \hat{\delta}^{*}}}^{1}\left(2 x-\frac{3}{2}\right) N x^{N-1} d x=2 \theta \widehat{\delta}^{*}
$$

Numerical computations show that $\widehat{\delta}^{*}>\delta^{*}$ is increasing $N$. This effect then makes $x^{R P}$ (and in this case also $v^{R P}$ ) increase in $N$. For example, when $\theta=\frac{1}{6}$.

\begin{tabular}{c|ccccccc}
$N$ & 4 & 5 & 6 & 7 & 8 & 9 & 10 \\
\hline$\delta^{*}$ & 0.3000 & 0.5000 & 0.6429 & 0.7500 & 0.8333 & 0.9000 & 0.9545 \\
$\widehat{\delta}^{*}$ & 0.3000 & 0.5034 & 0.6490 & 0.7552 & 0.8369 & 0.9023 & 0.9560 \\
$x^{R P}$ & 0 & 0.2534 & 0.3648 & 0.4190 & 0.4513 & 0.4729 & 0.4885 \\
$v^{R P}$ & 0 & 0.3758 & 0.4123 & 0.4388 & 0.4592 & 0.4756 & 0.4890
\end{tabular}


Notice that in this example, the formal reserve price, $v^{R P}$, is increasing in the number of bidders, though this may not necessarily be the case ${ }^{6}$.

\section{Conclusions}

Using a highly tractable model of information disclosure we have found that when the seller chooses how much (costly) information to provide and uses an optimal selling mechanism, he provides more information that when the object is always sold and the reserve price is no longer independent of the number of bidders. In particular, more bidders will lead the auctioneer to increase the amount of information provided and impose a more restrictive reserve price. Also, we find that while with few bidders an auctioneer that must always sell the good may not provide any information, if allowed to use a reserve price, the auctioneer may prefer to disclose some information and fix a reserve price, so that with positive probability the object may not be sold.

The model is standard (and general) in all dimensions but in the choice of the set of available signals. Our strategy of proof uses the linearity of our chosen information structures and it cannot be directly applied to a fully general set of signals. However, we trust that our results hold more generally since the intermediate steps have clear economic intuitions.

\footnotetext{
${ }^{6}$ Notice that $\frac{\partial v^{R P}}{\partial \delta}=\left(x^{R P}-v_{m}\right)+\frac{\partial x^{R P}}{\partial \delta}$. If $x^{R P}<v_{m}$, the first term is negative and the formal reserve price $v^{R P}$ may decrease in $\delta$.
} 


\section{REFERENCES}

[1] Bergemann, D. and Pesendorfer, M. (2007) "Information structures in optimal auctions," Journal of Economic Theory, Elsevier, vol. 137(1), pages 580-609, 2007.

[2] BoArd, S. (2009)"Revealing Information in Auctions: The Allocation Effect," Economic Theory 38(1), pp. 125-135, 2009.

[3] Eso, P. and Szentes, B. (2007) "Optimal Information Disclosure in Auctions and The Handicap Auction", Review of Economic Studies, 74, 705-731.

[4] Ganuza, J-J. (2004) "Ignorance promotes competition. An auction model of endogenous private valuations", forthcoming in Rand Journal of Economics, 35, 3, 583-598.

[5] Ganuza, J-J. and Penalva, J. (2010) "Signal ordering based on dispersion and the supply of private information in auctions", Econometrica, 78, 3, 1007-1030.

[6] Hummel, P. and McAfee, P. (2012) "When does improved targeting increase revenue", mimeo. 


\section{A Appendix}

\section{Proof of Lemma 1:}

(a) To prove that the virtual valuation function is increasing we just need to show that the slope of the inverse hazard rate of $G(\cdot, \delta)$ is negative. Recall that

$$
\frac{1-G(v, \delta)}{g(v, \delta)}=\delta \times \frac{1-F\left(\frac{v-(1-\delta) v_{m}}{\delta}\right)}{f\left(\frac{v-(1-\delta) v_{m}}{\delta}\right)}
$$

As the inverse hazard rate of $F$ is decreasing, and $\left(v-(1-\delta) v_{m}\right) / \delta$ is increasing in $v$, applying the chain rule it follows that the inverse hazard rate of $G$ will be decreasing.

(b) Let $S(v) \equiv \int_{0}^{v} G(s, \delta)-G\left(s, \delta^{\prime}\right)$ d $s . G\left(v, \delta^{\prime}\right)$ second order stochastically dominates $G(v, \delta)$ iff $S(v) \geq 0$ for all $v \in[0,1]$. Clearly, $S(0)=0$ and $S(1)=0$. Consider the slope of $S$ :

$$
\begin{aligned}
S^{\prime}(v) & =G(v, \delta)-G\left(v, \delta^{\prime}\right) \\
& =F\left(\frac{v-(1-\delta) v_{m}}{\delta}\right)-F\left(\frac{v-\left(1-\delta^{\prime}\right) v_{m}}{\delta^{\prime}}\right)
\end{aligned}
$$

As $F$ is monotone, $F\left(\frac{v-(1-\delta) v_{m}}{\delta}\right)-F\left(\frac{v-\left(1-\delta^{\prime}\right) v_{m}}{\delta^{\prime}}\right)>0$ iff $\frac{v-(1-\delta) v_{m}}{\delta}-\frac{v-\left(1-\delta^{\prime}\right) v_{m}}{\delta^{\prime}}=\frac{\left(v-v_{m}\right)\left(\delta^{\prime}-\delta\right)}{\delta^{\prime} \delta}>0$. This implies $S^{\prime}(v)>0$ if $v<v_{m}$, and $S^{\prime}(v) \leq 0$ otherwise. So that $S(v) \geq 0$. See also, Ganuza and Penalva (2010) that shows that $G\left(v, \delta^{\prime}\right)$ dominates $G(v, \delta)$ according to the dispersive order.

\section{Proof of Lemma 2 AND Proposition 2:}

Using a change of variable we can rewrite the auctioneer's expected revenue as

$$
\int_{0}^{1}\left[x \delta+(1-\delta) v_{m}-\delta \frac{1-F(x)}{f(x)}\right] m_{f}(x) d x
$$

where, $m_{f}(x)$, is the density function of the first order statistic associated to the distribution function of the maximum of $N$ independent draws from the distribution $F(\cdot)$, and $\left[x \delta+(1-\delta) v_{m}-\delta \frac{1-F(x)}{f(x)}\right]$ is non-decreasing. 
Then, the optimal reserve price can be characterized by $x^{R P}$, where $x^{R P}$ is obtained from the expression:

$$
x^{R P} \delta+(1-\delta) v_{m}-\delta \frac{1-F\left(x^{R P}\right)}{f\left(x^{R P}\right)}=0,
$$

which, using the function $J$, is implicitly defined by the expression:

$$
J\left(x^{R P}\right)=x^{R P}-\frac{1-F\left(x^{R P}\right)}{f\left(x^{R P}\right)}=-\frac{(1-\delta)}{\delta} v_{m}
$$

Then, the reserve price, $v^{R P}$ is obtained from $x^{R P}$ :

$$
v^{R P}=x^{R P} \delta+(1-\delta) v_{m}
$$

Given that $J(x)$ is increasing and $-\frac{(1-\delta)}{\delta} v_{m}$ is increasing in $\delta$, the larger $\delta$ the larger $x^{R P}$. Notice that $x^{R P}$ will not be well defined when $J(0)=-\frac{1}{f(0)}>-\frac{(1-\delta)}{\delta} v_{m}$, since then $x-\frac{1-F(x)}{f(x)}$ is always larger than $-\frac{(1-\delta)}{\delta} v_{m}$. In that case, $x^{R P}=0$, which is equivalent to not using a reserve price. Summarizing,

$$
x^{R P}=\left\{\begin{array}{cc}
J^{-1}\left(-\frac{(1-\delta)}{\delta} v_{m}\right) & \text { if } J(0)<-\frac{(1-\delta)}{\delta} v_{m} \\
0 & \text { Otherwise }
\end{array}\right.
$$

Finally, we can rewrite the condition $J(0)>-\frac{(1-\delta)}{\delta} v_{m}$ as a condition on $\delta$

$$
J(0)>-\frac{(1-\delta)}{\delta} v_{m} \Longleftrightarrow \delta>\bar{\delta}, \quad \text { where } \quad \bar{\delta}=\frac{v_{m}}{v_{m}+J(0)} .
$$

Proof of Proposition 3: The auctioneer's optimal amount of information, $\delta^{*}$, without a reserve price is given by this first order condition:

$$
\int_{0}^{1}\left[x-v_{m}-\frac{1-F(x)}{f(x)}\right] m_{f}(x) d x-C^{\prime}\left(\delta^{*}\right)=0
$$

Subtracting this equation from the first order condition of the auction with reserve price computed above,

$$
\frac{\partial \widehat{\pi}(\delta, N)}{\partial \delta}=\int_{x^{R P}}^{1}\left[x-v_{m}-\frac{1-F(x)}{f(x)}\right] m_{f}(x) d x-C^{\prime}\left(\widehat{\delta}^{*}\right)=0 .
$$

Taking the difference between the two F.O.C.s we obtain the following expression

$$
-\int_{0}^{x^{R P}}\left[x-v_{m}-\frac{1-F(x)}{f(x)}\right] m_{f}(x) d x=C^{\prime}\left(\widehat{\delta}^{*}\right)-C^{\prime}\left(\delta^{*}\right)
$$


The optimal $x^{R P}$ is obtained from the equation

$$
x^{R P} \delta+(1-\delta) v_{m}-\delta \frac{1-F\left(x^{R P}\right)}{f\left(x^{R P}\right)}=0 .
$$

Then,

$$
\begin{aligned}
v_{m}+\delta\left[x^{R P}-v_{m}-\frac{1-F\left(x^{R P}\right)}{f\left(x^{R P}\right)}\right] & =0 \\
\Rightarrow x^{R P}-v_{m}-\frac{1-F\left(x^{R P}\right)}{f\left(x^{R P}\right)} & =-\frac{v_{m}}{\delta} .
\end{aligned}
$$

As $x-v_{m}-\frac{1-F(x)}{f(x)}$ is monotone increasing this implies that the integral on the left hand side of Equation (5) is negative, so that the LHS of (5) is positive and $\widehat{\delta}^{*}>\delta^{*}$.

Proof of Proposition 4: We first establish that providing some information is optimal if $N$ is large enough. Consider the limit as $N \rightarrow \infty$ of $\widehat{\pi}(\delta, N)$. As $N$ goes to infinity, the distribution $m_{f}(x)$ converges to a mass point at $x=1$ so that $\lim _{N \rightarrow \infty} \widehat{\pi}(\delta, N)=v_{m}+\delta\left(1-v_{m}\right)-C(\delta)$. As $C^{\prime}(0)=0$, and $v_{m}<1$, the optimal $\widehat{\delta}^{*}$ will be positive. Consider that $\widehat{N}_{0}$ is the minimum $N$ such that it is optimal to provide information, then for $N>\widehat{N}_{0}$ it is optimal to do so too since $\widehat{\pi}(\delta, N)$ is increasing in $N$ for all $\delta, \widehat{\pi}\left(\widehat{\delta}^{*}, \widehat{N}_{0}\right)>v_{m} \Rightarrow \widehat{\pi}\left(\widehat{\delta}^{*}, N\right)>v_{m}$.

Monotonicity of $\widehat{\delta}^{*}(N)$ requires that $\forall \delta^{\prime} \in \widehat{\delta}_{N+1}^{*}, \forall \delta^{\prime \prime} \in \widehat{\delta}_{N}^{*}, \max \left\{\delta^{\prime \prime}, \delta^{\prime}\right\} \in \delta_{N+1}^{*}$ and $\min \left\{\delta^{\prime \prime}, \delta^{\prime}\right\} \in$ $\delta_{N}^{*}$. Then, if $\delta^{\prime} \in \widehat{\delta}_{N+1}^{*}$, we want to rule out that there exists a $\delta^{\prime \prime} \in \delta_{N}^{*}, \delta^{\prime \prime}>\delta^{\prime}$ such that either (a) $\delta^{\prime} \notin \delta_{N}^{*}$, i.e. $\widehat{\pi}\left(\delta^{\prime \prime}, N\right)-\widehat{\pi}\left(\delta^{\prime}, N\right)>0$ and $\widehat{\pi}\left(\delta^{\prime \prime}, N+1\right)-\widehat{\pi}\left(\delta^{\prime}, N+1\right) \leq 0$, or $/$ and (b) $\delta^{\prime \prime} \notin \widehat{\delta}_{N+1}^{*}$, i.e. $\widehat{\pi}\left(\delta^{\prime \prime}, N\right)-\widehat{\pi}\left(\delta^{\prime}, N\right) \geq 0$ and $\widehat{\pi}\left(\delta^{\prime \prime}, N+1\right)-\widehat{\pi}\left(\delta^{\prime}, N+1\right)<0$. We rule out both cases by showing that for $\delta^{\prime} \in \widehat{\delta}_{N+1}^{*}$ and all $\delta^{\prime \prime}>\delta^{\prime}$

$$
\widehat{\pi}\left(\delta^{\prime \prime}, N+1\right)-\widehat{\pi}\left(\delta^{\prime}, N+1\right) \geq \widehat{\pi}\left(\delta^{\prime \prime}, N\right)-\widehat{\pi}\left(\delta^{\prime}, N\right) .
$$

This condition is equivalent to the following one:

$$
\int_{\delta^{\prime}}^{\delta^{\prime \prime}}\left[\frac{\partial \widehat{\pi}(\delta, N+1)}{\partial \delta}-\frac{\partial \widehat{\pi}(\delta, N)}{\partial \delta}\right] d \delta \geq 0
$$

We start by computing $\frac{\partial \widehat{\pi}(\delta, N)}{\partial \delta}$. Using a change of variable, we can write the auctioneer's profits as

$$
\widehat{\pi}(\delta, N)=\int_{x^{R P}}^{1}\left[x \delta+(1-\delta) v_{m}-\delta \frac{1-F(x)}{f(x)}\right] m_{f}(x, N) d x-C(\delta)
$$


where $m_{f}(x, N)$ is as defined above, only now we make the dependence on $N$ explicit. Note that $\widehat{\pi}$ is continuously differentiable in $\delta$ so that condition (6) is well-defined.

Consider the first order condition of the problem-we can safely ignore the effect of $\delta$ on $\widehat{\pi}$ via $x^{R P}$ because if there is no reserve price $\left(x^{R P}=0\right)$ the derivative is cero and if there is one, it is set optimally, and the derivative of $x^{R P}$ with respect to $\delta$ will be cero by the envelope theorem.

$$
\frac{\partial \widehat{\pi}(\delta, N)}{\partial \delta}=\int_{x^{R P}}^{1}\left[x-v_{m}-\frac{1-F(x)}{f(x)}\right] m_{f}(x) d x-C^{\prime}\left(\delta^{\hat{A}}\right)
$$

This expression can be rewritten equivalently as follows:

$$
\frac{\partial \widehat{\pi}(\delta, N)}{\partial \delta}=\left(P^{*}(\delta, N)-v_{m}\right)\left(1-M_{f}\left(x^{R P}(\delta), N\right)\right)-C^{\prime}(\delta),
$$

where $P^{*}(\delta, N)=E_{\delta, N}\left[x_{1}-\frac{1-F\left(x_{1}\right)}{f\left(x_{1}\right)} \mid x_{1} \geq x^{R P}\right]$ is the expected value of the virtual valuation of the first order statistic of the signal, conditioned on the event that the highest realization of the signal is greater than the signal corresponding to the reserve price $\left(x^{R P}\right)$. The second term, $\left(1-M_{f}\left(x^{R P}(\delta), N\right)\right)$, is the probability of selling the good, which is equivalent to the probability that the highest realization of the signal is larger than the reserve price $x^{R P}(\delta)$.

A well established property of $m_{f}$ is that the family of distributions $\left\{m_{f}(x, N)\right\}_{N}$ indexed by $N$ is increasing in the likelihood-ratio order. This implies that $m_{f}(x, N+1)$ is (first-order) stochastically greater than $m_{f}(x, N)$ on any interval of the form $[x, 1]$. Thus, $\left(1-M_{f}\right)$ is increasing in $N$. As by assumption $x-\frac{1-F(x)}{f(x)}$ is an increasing function of $x$, then $P^{*}$ is also increasing in $N$. Moreover, $P^{*}$ is increasing in $\delta$ since $x^{R P}(\delta)$ is also increasing in $\delta$.

From our premise, $\delta^{\prime} \in \widehat{\delta}^{*}(N+1)$, which implies $P^{*}\left(\delta^{\prime}, N+1\right)>v_{m}$. We want to show that $\frac{\partial \widehat{\pi}(\delta, N+1)}{\partial \delta}-\frac{\partial \widehat{\pi}(\delta, N)}{\partial \delta} \geq 0$ for all $\delta \in\left[\delta^{\prime}, \delta^{\prime \prime}\right]$. For $\delta \in\left[\delta^{\prime}, \delta^{\prime \prime}\right]$ there are two possibilities: (1) if $P^{*}(\delta, N) \leq v_{m}$, then $\frac{\partial \widehat{\pi}(\delta, N+1)}{\partial \delta}-\frac{\partial \widehat{\pi}(\delta, N)}{\partial \delta} \geq 0$ is immediate as $P^{*}\left(\delta^{\prime}, N+1\right)>v_{m}$ and $P^{*}$ is increasing in $\delta$. (2) If $P^{*}(\delta, N)>v_{m}$, we know from above that $P^{*}(\delta, N)$ and $1-M_{f}$ are increasing in $N$, so that

$$
\left(P^{*}(\delta, N+1)-v_{m}\right)\left(1-M_{f}\left(x^{R P}(\delta), N+1\right)\right) \geq\left(P^{*}(\delta, N)-v_{m}\right)\left(1-M_{f}\left(x^{R P}(\delta), N\right)\right)
$$

Thus, $\delta^{\prime} \in \widehat{\delta}^{*}(N+1)$ implies that for $\delta^{\prime \prime}>\delta^{\prime}$

$$
\widehat{\pi}\left(\delta^{\prime \prime}, N+1\right)-\widehat{\pi}\left(\delta^{\prime}, N+1\right) \geq \widehat{\pi}\left(\delta^{\prime \prime}, N\right)-\widehat{\pi}\left(\delta^{\prime}, N\right) .
$$


ii) Follows from $\widehat{\pi}(0, N)=\pi(0, N)=v_{m}$ and $\widehat{\pi}\left(\widehat{\delta}^{*}, N\right) \geq \pi\left(\delta^{*}, N\right)$. Then, $\pi\left(\delta^{*}, N_{0}\right)>v_{m}$ $\Rightarrow \widehat{\pi}\left(\widehat{\delta}^{*}, N_{0}\right)>v_{m}$ and it is possible that there exist $N$ such that $\pi\left(\delta^{*}, N\right)<v_{m}<\widehat{\pi}\left(\widehat{\delta}^{*}, N\right)$, meaning $\widehat{N}_{0} \leq N_{0}$.

Proof of Collorary 5: Inmediate from the result i) in Proposition 4 and results ii) and iii) in Proposition 2. 